\title{
- J oint Venture: Estrategia para LograR la Competitividad Empresarial en el Perú
}

(1) Raúl Maguiña F.

\section{RESUMEN}

El artículo propone una estrategia de competitividad empresarial utilizando el joint venture, a partir de alianzas que junten unidades de negocio, analizándose el proceso de su creación con los requerimientos de compromiso transparente y de legalidad, dentro del marco legal peruano. A fin de lograr la factibilidad de diversos proyectos de inversión, en cualquiera de los sectores productivos del País.

Palabras Claves: Globalización. Innovación tecnológica. Joint Venture. Competitividad empresarial.

J ant Venture: Strategy to Obtain the Managerial Competitiveness in Peru ABSTRACT

This article proposes a managerial competitiveness strategy using the JointVenture enterprise, rising out of alliances linking business units, by analizing its making-up process through legal and clear compromise requirements, framed within the peruvian law, and with the purpose of getting feasibility of various investment projects, in any production sector in our country.

Key Words: Globalization. Technological innovation. Joint venture. Managerial competitiveness.

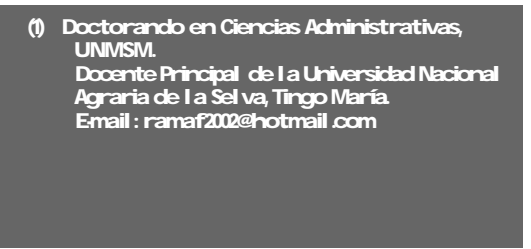

\section{INTRODUCCIÓN}

En el proceso de la globalización de la economía y de creación de mercados mundiales, se produce un flujo creciente de inversiones internacionales de los países productores de bienes de capital hacia países con vastos recursos naturales o de mercados propios interesantes o que disponen de una mano de obra calificada y poco costosa .

Existen muchas razones y consideraciones político-legales, económicas y ventajas comparativas y competitivas, para decidir las inversiones, que se multiplican rápidamente. Gran parte del capital de inversión no se efectúa de manera directa, sino por el método de las Alianzas Estratégicas, utilizando el mecanismo de Joint Venture como el instrumento internacional típico para concretar dichas inversiones.

Los Joint Venture son dentro de esta concepción, una forma de alianza estratégica que se ha manifestado con total pujanza en el Perú en los "procesos de privatización", lo que en su casi totalidad se concretaron por medio de empresas regularmente constituidas en el país; pero la realidad económica y jurídica es que dichas empresas se han alimentado de acuerdos de Joint Venture, que presentan los caracteres de una alianza contractual y temporaria concreta, en la que los inversores extranjeros han buscado alianzas con capitales nacionales para tomar participaciones en mercados hasta ahora desconocidos. Esas mismas posibilidades se abren frente al desafio que mediante el TLC están por concretarse.

Al respecto, de estas negociaciones existe una ironía que al final termina por manifestarse (así lo manifiesta el alemán Wirth), «los Joint Venture son una operación en que el extranjero aporta su dinero y el socio local su conocimiento del país, y al cabo de algunos años resulta que el socio local detenta el dinero y extranjero ha aprendido a conocer el País».

Así mismo, se debe recordar que el sector externo ha jugado un papel significativo en las economías y donde estas últimas buscan la integración económica continental e intercontinental, aunque su conceptualización e implementación está en proceso de reajuste y consolidación; debiendo entender además que el proceso de integración, que al reducir o eliminar la protección genera nuevas condiciones competitivas que implican una necesidad de ajuste, no solamente en la política económica sino en la toma de decisiones empresariales, que tendrán en el espacio ampliado un nuevo marco de oportunidades y restricciones.

Al respecto M. Porter, en su libro "Ventaja Competitiva de las Naciones" (1991), menciona que la prosperidad de una nación no es hereditaria, si 
no que se crea, y que depende de la capacidad de su sistema empresarial de innovar y mejorar. Asimismo, considera que las Alianzas Estratégicas son una forma de competir en el mercado para alcanzar y mantener una ventaja competitiva. La competencia futura ya no se dará entre empresas individuales, si no entre nuevas y complejas agrupaciones corporativas.

En ese sentido, el propósito central de este artículo es justamente describir los mecanismos de ajustes y de cooperación empresarial que son necesarios y convenientes en el marco de integración y así como el mecanismo de cooperación denominado "Joint Venture".

\section{J OINT VENTURE}

Ant ecedentes

El término Joint Venture proviene de Joint Adventure y su origen se remonta a las relaciones de derecho que surgieron de la ley de partnership hacia fines del siglo XIX.

Sin embargo, algunos autores mencionan que su origen es escocés y que se utilizaba un siglo antes. La polémica carece de relevancia. Lo cierto que es el gran salto y la utilización masiva de los joint ventures proviene de los EE. UU. en los que tienen gran repercusión, en virtud de un régimen fiscal que favoreció su crecimiento.

La expresión joint venture hace referencia a una modalidad de colaboración empresarial cuyo origen se sitúa en el Derecho angloamericano. Nacida a finales del siglo pasado en los Estados Unidos y desarrollada en la primera mitad del siglo XX en aquel país, ha ido adquiriendo relevancia en el tráfico internacional a lo largo de las últimas décadas y, en consecuencia, ha llegado también a ponerse en práctica de América Latina.

Las palabras Joint Venture aparecen en la actualidad en todos los medios de difusión de conocimiento y de información. En los años 60', constituía un concepto nuevo cuyos alcances no estaban bien delimitados ni comprendidos. Desde los años 70' empezó una corriente de interés, que no se ha detenido hasta ahora, aunque sus implicancias legales varíen de acuerdo con el lugar donde se hace Joint Venture. En cualquier caso resulta atinado puntualizar que en los últimos años, su desarrollo ha sido enorme, y se constituye como parte de las opciones que las empresas tienen a su disposición al momento de toma de decisiones de inversión en el mundo local o en el ámbito internacional.
Al respecto se puede decir que, las estrategias de internacionalización de las empresas a través de Joint Venture, están siendo utilizadas en forma creciente debido a otro aspecto importante de la economía internacional: la formación de bloques económicos. En efecto esto lleva a los empresarios a constituir empresas conjuntas para no quedar fuera del terreno de los negocios.

Finamente, tras la fase de internacionalización quedaron algunos rasgos, los más característicos: Flexibilidad, control conjunto, y deber de fidelidad.

Def inición

El Joint Venture, es también conocido como empresa de riesgo compartido, empresa con participación, empresa conjunta o co-inversión de riesgo.

Es una forma de cooperación empresarial en un contexto competitivo que actúa como una "asociación empresarial estratégica" entre dos o más empresas nacionales y/o extranjeras, que mediante la integración, interacción y complementariedad de sus actividades y recursos buscan alcanzar propósitos comunes.

El Joint Venture ha adquirido un desarrollo sorprendente, al punto que se constituye como parte de las opciones que las empresas tienen para tomar decisiones de inversión en el ámbito local o internacional; para lograr sinergias y los beneficios mutuos, y compartiendo los riesgos; variando sus características de acuerdo al tipo de negocio y país en que se ubica.

Q asificación y Tipol ogía

a. Por su modalidad legal y/u organizacional

1. Joint Venture Societaria: Es una organización empresarial que da origen a una nueva entidad o a una nueva sociedad con las implicancias jurídicas que ello significa por su rigidez y complejidad con la diversidad de las leyes de cada país. Está ligada al sistema jurídico del "Civil Law".

2. Joint Venture Contractual: Consiste en una relación contractual que no da origen a una nueva entidad jurídica. Pragmatismo, flexibilidad, autonomía, son características inherentes a este Joint Venture. Está ligada al sistema jurídico del "Common Law".

\section{b. Por su contexto geográfico}

1. Joint Ventures Nacional: Son las creadas por socios con idéntica nacionalidad y dentro de su país de origen.

2. Joint Venture Internacional: Son las creadas por socios de diferentes nacionalidades. Se consi- 
dera como socio local el que tenga el domicilio social en el país donde se instale la Joint Venture.

\section{c. Por el tipo de contrato o acuerdos de las partes}

1. Para proyectos manufactureros.

2. Para las industrias extractivas.

3. Para la industria de la construcción.

4. Para proyectos comerciales.

5. Para investigación y desarrollo.

6. Para actividades financieras.

7. Para prestación de servicios.

8. Para actividades de turismo.

9. Paraactividades agropecuarias yagroindustriales. 10.Otros

\section{d. Por el rol o desempeño de los socios}

1. Joint Venture con un Socio Dominante: Significa que el emprendimiento está básicamente controlado o dominado por el socio, que juega un rol activo, mientras que el otro socio tiene un rol pasivo.

2. Joint Venture de Administración y Operación Compartida: Significa que ambos socios juegan un rol activo en la administración y gerenciamiento de la empresa.

3. Joint Venture Independientes: Significa que ninguno de los socios juega un rol activo. El papel fundamental en el proceso de toma de decisiones, y en la administración y operación de la empresa o el proyecto, recae en manos de un gerente general, que habitualmente no proviene de ninguno de los socios.

Proceso de For mación de un Joint Venture El proceso de formación de un Joint Venture es complejo y pasa por varias etapas a saber:

a. Identificación de objetivos.

b. Selección del socio o socios venturistas.

c. Intercambio de información confidencial.

d. Preparación de una carta de intención

e. Elección de la forma del negocio.

f. Identificación de los problemas entre socios.

g. Redacción de convenio de Joint Venture.

Menciona Colaiacovo que el esquema de formación de un Joint Venture tiene los siguientes pasos:

a. Contactos, conversaciones y negociaciones.

b. Protocolo de intención.

c. Acuerdo base.

d. Contratos satélites (formación de empresa, acuerdos de accionistas).

Estos diferentes pasos se pueden sintetizar en la práctica en conversaciones preliminares, acto de intención sobre la posibilidad de su futuro negocio, convenido de Joint Venture propiamente dicho y acuerdos complementarios.
En el Acuerdo de Intenciones, comúnmente se fijan: a. Descripción de objetivos comunes.

b.Acuerdo sobre realización de un estudio de factibilidad.

c. Aportes de información de cada participante.

d. Compromiso de confidenciabilidad recíproco y por un plazo variable, que puede abarcar la duración total del proyecto.

e. Plazo para tomar decisiones, luego de concreción y entrega del acuerdo de factibilidad.

f. Ausencia de responsabilidad de los partícipes por el abandono del proyecto, como o sin motivo.

g. Explicación de gastos comunes y propios de cada firmamento.

h. Renuncia a reclamos por gastos unilaterales en demasía.

Car acter ísticas Principal es del Joint Venture

a. Autonomía.

b. Pluralidad de prestaciones.

c. Consensualidad.

d. Informalidad.

e. Ejecución continuada o periódica.

f. Atipicidad.

g. Naturaleza asociativa.

h. Relación de lealtad.

i. Ausencia de personería jurídica.

j. Flexibilidad.

k. Onerosidad.

I. Contribución de los ventures.

m.Beneficio económico común.

Inter és de I os Países por I os Joint Venture

Actualmente, dada las ventajas que representa sumar esfuerzos y fortalezas para lograr la competitividad, los países han mostrado interés por este sistema por lo siguiente:

a. Acceso al capital internacional.

b. Acceso a nuevas tecnologías, conocimientos, patentes y marcas.

c. Aumento del proceso productivo y la consecuente generación de empleo.

d. Acceso al mercado internacional, a través del socio extranjero.

e. Aumento de la exportaciones, creando el incremento de divisas.

f. Sustitución de importaciones.

g. Mejoramiento de las capacidades de gestión y cultura empresarial .

h. Mejoramiento de la capacidad de I + D (+ I).

i. Creación de infraestructura social.

j. Dinamicidad o mejora de la economía nacional.

k. Competitividad del sistema empresarial nacional

Motivos par a I a Formación de un Joint Venture La Figura 1 muestra los motivos para la formación de un Joint Venture. 


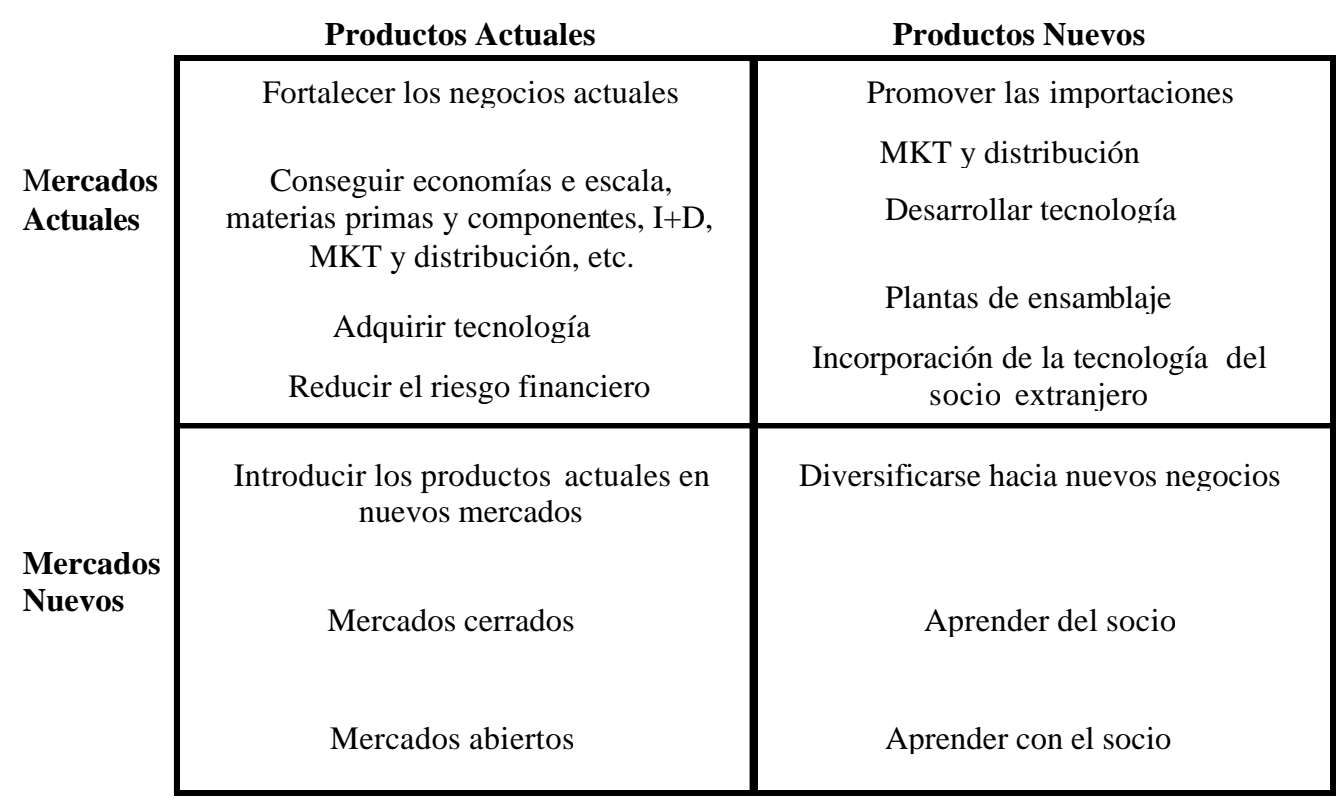

Figura 1. Motivos paralaformaciónde un JointVenture

Los Joint Vent ure en América Lat ina

En América Latina hasta la fecha y en un alto porcentaje, la especialización exportadora responde al aprovechamiento de la ventaja comparativa estática en la mayoría de los casos.

La teoría económica tradicional estableció a partir de Bela Balassa (1964), las etapas en dirección a formas más completas de integración.

1. Área de Libre Comercio. Se busca la libre circulación de bienes dentro de la región.

2. Unión Aduanera. Además de las características anteriores, los países miembros buscan una estructura uniforme de barreras tarifarias en relación al resto del mundo.

3. Mercado Común. Al interior, se agrega la libre circulación de mano de obra y capitales, esto es la libre circulación de bienes y factores de producción.

4. Unión Económica. Es la etapa superior del proceso de integración donde a las características del punto anterior se suma con alto grado de integración y coordinación de las políticas macroeconómicas, en especial la monetaria y fiscal.

La principal motivación para la integración -aparte de los aspectos políticos- es la perspectiva de un beneficio económico a través de :

a. Crecimiento de la producción en función de la especialización con menores costos, como consecuencia de las economías de escala;

b. Aumento de la eficiencia, a consecuencia de la com- petencia interna, lo que conllevaría a mejorar los términos de intercambio con el resto del mundo; $y$

c. Alteraciones estructurales inducidas por la integración, afectando la cantidad y calidad de los insumos de factores tales como flujos de capitales y avances tecnológicos.

Sin embargo, a pesar de que los países muestran interés para que cada vez su sistema empresarial se vuelva más competitivo a través de la constitución de Joint Venture, existen ciertos factores desde el punto de vista de estructura gubernamental que limitan su desarrollo. Estos inconvenientes son:

a. Falta de consistencia en las reglamentaciones que regulan la inversión extranjera.

b. Procesos administrativos engorrosos y burocráticos para las autorizaciones de la constitución de la inversión extrajera.

c. Política crediticia y financiera inadecuada

d. Abrumadora carga tributaria y en muchos casos anti-técnicos.

e. Existencia de un mercado informal creciente

f. Infraestructura básica inadecuada (ejemplo: transporte, zonas industriales).

Y para contrarrestar lo anterior la mayoría de los países sudamericanos tienes que revertir muchos aspectos que conlleven a la presentación de los siguientes incentivos:

a. Incentivos legales

b. Incentivos financieros

c. Incentivos no financieros 
Otros puntos de igual importancia, y muchas veces también son decisivos para que el capital extranjero ancle en nuestro país, es trabajar para crear un clima de confianza y para ellos se deberá establecer una:

- Estabilidad política

- Estabilidad jurídica

- Estabilidad social

\section{LOS JOINT VENTURE EN EL PERÚ}

Nuestro país, aún con sus dificultades políticas, sociales y económicas, constituye un escenario importante para hacer negocios. Pero este sistema del Joint Venture no está muy difundido, principalmente por la falta de una visión empresarial competitiva.

A continuación se muestran las prioridades de las Empresas Peruanas para formar un Joint Venture $1^{\circ}$ Inversión extranjera en operaciones.

$2^{\circ}$ Penetración de mercados y cobertura más competitiva. $3^{\circ}$ Inversión en I + D para desarrollar nuevos productos. $4^{\circ}$ Mejorar la capacidad productiva para el comercio exterior.

$5^{\circ}$ Desarrollo de productos.

$6^{\circ}$ Acceso a ventajas tributarias.

$7^{\circ}$ Incorporación de nuevas tecnologías y know how de gestión.

$8^{\circ}$ Incremento de rentabilidad.

$9^{\circ}$ Participación conjunta de capitales en empresa común.

Lamentablemente, en nuestro país no existe estudios o estadística de las empresas que han hecho o están haciendo Joint Venture, tal vez puede ser porque este tipo de negociación no se registra en ninguna entidad, ya que todos saben que los Joint Venture es un contrato contractual que no esta obligado a crear una nueva sociedad (que lo haría visible) y menos registrarlo. Indudablemente esto hace que no se pueda saber con certeza el impacto que tienen los Joint Venture en el desarrollo del país.

\section{SISTEMA LEGAL DEL J OINT VENTURE EN EL PERÚ}

Joint Vent ure y I a Ley General de Sociedades La inclusión de los contratos asociativos en esta Ley constituye un tema controvertido. Algunos consideran que los contratos de colaboración empresarial deben ser regulados por esta ley, en tanto se precisa que sí habían sido considerados por la anterior Ley de Sociedades Mercantiles (1996).

Otras posiciones académicas señalan que la Ley General Sociedades no debe regular contratos que no dan lugar a nacimiento de persona jurídica, y que podrían llevar a confusiones entre la naturaleza jurídica de las sociedades y de los contratos. Además, que el Joint Venture es un contrato innominado y atípico.

Existen posiciones fundamentadas que la regulación del Joint Venture no es necesaria, porque son las partes quienes dotan al contrato de contenido, características, obligaciones y derechos, por lo que su regulación debe ser por el Código de Comercio o Código de la Empresa

En el Perú existen normas legales que hacen referencia a este contrato :

* Decreto Supremo № 010 - 88 - PE (23-03-88) que introduce por primera vez el término Joint Venture.

* Decreto Legislativo N 662 (02-09-91), Ley de Promoción de la Inversión Extranjera.

* Reglamento de la Ley de impuesto a las Ventas e Impuesto Selectivo al Consumo.

* Decreto Legislativo № 674 (27-09-91), Ley de Crecimiento de la Inversión Privada.

* Decreto Legislativo № 708 (14-11-91), Ley de Promociones de Inversiones en el Sector Minero.

* Decreto Legislativo No 757 (13-11-91), Ley Marco para el Crecimiento de la Inversión Privada, que establece garantías aplicables a los que concurren en una co-inversión.

* Decreto Supremo Nº 014 - 92 - EM (04-06-92), que incluye normas referentes al contrato de riesgo compartido.

* Decreto Supremo No 162 - 92-EF (12-10-92), Reglamento de los Decreto Legislativo 662 y 757.

El anterior Marco Jurídico, es complementado con Convenios Multilaterales, tales como el Convenio MIGA (Acuerdo de Garantías de Inversiones Multilaterales Diciembre de 1990) y el Convenio OPIC (Corporación Privada de Inversión en Otros Países, Noviembre de 1992).

Igualmente cumplen el mismo fin los Tratados Bilaterales con Suiza, Tailandia, Alemania, Corea del Sur, Países Bajos, Suecia, Reino Unido, Italia, Francia, Canadá, Chile y Argentina entre otros.

Es importante mencionar las preferencias arancelarias que, en un régimen de comercio exterior, ofrecen EE. UU., Japón y Europa. La Ley de Preferencias Comerciales Andinas (ATPA), permite el ingreso a EE. UU. De más de 6,100 productos. El Sistema General de Preferencias de la Comunidad Europea (SGP-CE) a los países andinos.

Aquí también están las diversas integraciones para sacar provecho con los JV: NAFTA (Acuerdo de Libre 
Comercio de América del Norte), APEC (Consejo Económico Asia-Pacífico), CAN (Comunidad Andina), MERCOSUR, ALCA (Acuerdo de Libre Comercio de las Américas), y el futuro TLC (Tratado de Libre Comercio) con EE. UU.

\section{CONCLUSIONES}

El Joint Venture es una forma asociativa que no requiere la creación de un sujeto de derecho. Nació libre, no sujeto a una forma reglada, es parte de la maravillosa tradición creativa del "common law", de la institución creada por la autonomía de la voluntad y reconocida en derecho por el juez legislador del common law.

No existe estadística ni estudios de Joint Venture en el Perú. Lo que dificulta el aprovechamiento de las ventajas comparativas y la maximización de las oportunidades del mercado.

Los Joint Venture responden mejor y más eficazmente a los contextos micro y macro económicos actuales y de futuro (asumiendo las tendencias mundiales y nacional actual). Por lo tanto, la constitución de Joint Venture en el Perú es limitada, en primer lugar por el desconocimiento o falta de visión competitiva del empresariado nacional

\section{BIBLIOGRAFÍA}

1. Arotoma Cacnahuaray, Sixto. (1999). Factibilidad Operativa de Joint Venture en la Producción y Comercialización de Frijol: Perú. Tesis para optar el Grado de magíster en Administración, mención Comercio Internacional, FCA -UPG, UNMSM, Lima Perú.

2. Bravo Melgar, Sydney Alex. (2003). Contratos Atípicos e Innominados, Contratos Modernos Empresariales. Ediciones Legales Iberoamericanas, Lima Perú.

3. Buchel, Bettina. (2002). La Búsqueda del Equilibrio de la Gestión de Joint Venture. Harvard Deusto Bussiness Review, Bilbao, Marzo del 2002.
4. Colaiacovo, Juan Luis; Avaro, Ruben Daniel y Otros. (1992). Joint Venture y Otras Formas de Cooperación Empresaria Internacional. Ediciones MACHI, Argentina.

5. Fernández Sánchez, Esteban. (1996). Innovación, Tecnología y Alianzas Estratégicas. Editorial Civitas S. A., España.

6.Lucich, Ivan M. (1994). Joint Venture en Busca de Competitividad. Revista $1 / 2$ de Cambio, Lima, Mayo de 1994.

7. Maguiña Flores, Raúl. (2003). La Globalización. Editorial Universidad Nacional Agraria de la Selva, Tingo María, Perú.

8. Malca Guaylupo, Oscar. (1995). Los Joint Venture y la Promoción de Exportaciones. Revista Perú Exporta, №224, Lima, Abril de 1995.

9. Marzorati, Osvaldo J. (1996). Alianzas Estratégicas y Joint Venture. Editorial ASTREA, Argentina.

10. Miquel Rodríguez, Jorge. (1998). La Sociedad Conjunta (Joint Venture Corporación). Editorial Civitas S. A., España.

11. Miranda Alcántara, Manuel Ivan. (2003). El Joint Venture en la Ley General de Sociedades. Revista Jurídica del Perú, Año LIII, N50, Setiembre del 2003, Lima Perú.

12. Porter, Michael E. (1991). La Ventaja Competitiva de las Naciones". Ediciones Vergara, Argentina.

13. PETROPERU (2004). Entrevista al Presidente del Directorio, Dr. Alejandro Narváez Liceras, "Interés de Empresas en hacer Joint Venture con Petro Perú". Lima Perú.

14. Sierralta, Aníbal. (1997). Joint Venture Internacional. $2^{\circ}$ edición, Editorial Pontificia Universidad Católica del Perú, Lima Perú.

15. Velásquez, Francisco. (1997). El Contrato de Joint Venture. Editorial Lexis Nexis, Santiago de Chile, Chile. 